Relations industrielles

Industrial Relations

\title{
Inflation et fiscalité
}

\section{Inflation and Fiscal Policy}

\section{André Raynauld}

Volume 30, numéro 3, 1975

URI : https://id.erudit.org/iderudit/028626ar

DOI : https://doi.org/10.7202/028626ar

Aller au sommaire du numéro

Éditeur(s)

Département des relations industrielles de l'Université Laval

ISSN

0034-379X (imprimé)

1703-8138 (numérique)

Découvrir la revue

Citer cet article

Raynauld, A. (1975). Inflation et fiscalité. Relations industrielles / Industrial Relations, 30(3), 299-318. https://doi.org/10.7202/028626ar
Résumé de l'article

Nous vivons dans une inflation, qui apparaît de plus en plus comme étant la fille naturelle de la croissance, sa seconde nature. Dans ce contexte, l'analyse des politiques de l'après-guerre montre la nécessité d'avoir recours à de nouveaux instruments.
Tous droits réservés @ C Département des relations industrielles de l'Universite Laval, 1975
Ce document est protégé par la loi sur le droit d'auteur. L’utilisation des services d'Érudit (y compris la reproduction) est assujettie à sa politique d'utilisation que vous pouvez consulter en ligne.

https://apropos.erudit.org/fr/usagers/politique-dutilisation/ 


\section{Inflation et fiscalité}

\section{André Raynauld}

Nous vivons dans une inflation, qui apparaît de plus en plus comme étant la fille naturelle de la croissance, sa seconde nature. Dans ce contexte, l'analyse des politiques de l'après-guerre montre la nécessité d'avoir recours à de nouveaux instruments.

En 1936, dans un ouvrage qui fait date et dont le titre - Prospérité et Dépression - est révélateur des préoccupations de l'époque des années 30 , Haberler définissait la pierre philosophale des politiques économiques, l'objectif sacro-saint de tous les gouvernements : assurer simultanément la croissance, le plein emploi et la stabilité des prix. Les trois conditions de la croissance équilibrée constituaient autant de sommets d'un triangle que les instruments de politiques économiques devaient permettre de tracer.

L'histoire économique de l'après-guerre s'analyse comme une course sans fin vers cet objectif, toujours assoupli au fil des années, mais jamais assez semble-t-il pour qu'il puisse être atteint de façon durable.

Haberler avait défini les objectifs mais pas les moyens. Très vite on se rendit compte que fermer le triangle était un casse-tête que l'on ne parvenait pas à résoudre, mais que par contre on arrivait assez bien à atteindre deux des objectifs sur trois. Croissance et plein tmploi étaient le plus facile, mais les prix dérapaient. Croissance et stabilité des prix allaient de pair pourvu que l'expansion fut modérée, mais le niveau d'emploi en souffrait un peu. Il semblait vain de vouloir concilier plein emploi et prix stables, et le triangle d'Haberler devenait quelque chose de chimérique.

On assouplit donc la règle notamment aux États-Unis et au Canada en définissant le plein niveau d'emploi comme étant celui qui correspondait à un taux de chômage de $4 \%$, juste un point de plus que le fameux $3 \%$ de Lord Beveridge en 1944. Tout allait devenir plus facile d'autant que les praticiens bénéficièrent du soutien de la théorie, quand l'économiste anglais Phillips traça

RAYNAULD, A., président, Conseil économique du Canada, Ottawa. 
en 1958 quelques courbes devenues célèbres depuis, semblant démontrer l'existence d'une relation étroite et stable entre le rythme de hausse de prix et le taux de chômage. Quand le taux de chômage s'abaissait, les prix augmentaient et vice versa. De plus, lorsque l'on s'approchait $\mathrm{du}$ niveau de plein emploi, on devait pour réduire davantage le taux de chômage, accepter des augmentations plus que proportionnelles des prix. Tout devenait donc clair, la règle d'Haberler était tout simplement excessive. L'analyse justifiait son abandon partiel, et par surcroit, elle offrait aux pouvoirs publics un instrument apparamment efficace leur permettant de procéder aux ajustements nécessaires, selon qu'ils accordaient la priorité à la stabilité des prix ou à l'emploi. Par ailleurs, l'évidence même leur permettait de pouvoir compter sur une autre liaison tout aussi stable entre croissance et emploi.

Le triangle est donc transformé et au cours des années 60 , la règle devient celle des trois fois $3 \%$. On parvient assez bien à faire coincider un minimum de croissance en volume de $3 \%$ avec une hausse maximum des prix de $3 \%$ pour un taux de chômage de l'ordre de $3 \%$. On n'en élimine pas pour autant les cycles économiques mais ceux-ci sont fortement atténués. La croissance ne fait le plus souvent que se ralentir, quitte parfois à ce que les pouvoirs publics provoquent un tel ralentissement lorsque l'économie a tendance à sortir du cadre des $3 \%$, pour ensuite repartir d'un meilleur pied. Mis à part les États-Unis et le Canada, chez qui le taux de chômage dépasse les $3 \%$ mais où les prix sont plus stables, la plupart des pays de l'OCDE ont à peu près tous dessiné convenablement cette nouvelle représentation du triangle. L'inflation existait, mais elle ne faisait que ramper.

Dans la deuxième moitié des années 60 , les choses vont moins bien et la règle des trois fois trois vole en éclats. La liaison prix-chômage se distend, on enregistre des phases de stagflation, l'inflation continue de ramper même en période de croissance ralentie et d'augmentation du chômage. C'est ainsi qu'aux États-Unis, on observe à la fin des années 60 , que pour un taux de chômage de l'ordre de $4 \%$, il faut dorénavant accepter un taux d'inflation de 1.5 point plus élevé qu'en 1950 pour obtenir un même résultat. En juin 1971, les experts du Comité de politique économique de l'OCDE écrivaient: "Il a été reconnu en fait, qu'il n'existait pas de relation simple ou constante, entre les rythmes de hausse des prix d'une part, et les degrés de pressions de la demande, les taux de croissance et les niveaux d'emploi de l'autre. \Diverses explications ont été proposées ajoute le Comité et «les avis ont différé du point de vue de savoir si l'on a affaire à un changement définitif ou à un phénomène 
temporaire ». La réponse a été donnée depuis par une explosion des prix que ne calment ni la montée du chômage, ni le ralentissement de la croissance. Il n'y a plus de triangle, les politiques ont perdu leur gyrocompas. La hausse des prix n'a cessé de s'accélérer depuis 1969 quels que soient les niveaux de chômage et les taux de croissance. En France, elle est passée de $5.3 \%$ en 1969 à $13.6 \%$ en 1974; en Allemagne, de $2.7 \%$ à $7 \%$; en Italie, de $1.4 \%$ à $19.1 \%$; en Grande Bretagne, de $5.5 \%$ à $16 \%$; en Belgique, de $3.8 \%$ à $12.6 \%$; en Suède, de $2.7 \%$ à $9.9 \%$; au Japon, de $5.2 \%$ à $24.4 \%$. Grâce à un léger ralentissement en 1970 et 1971 , le rythme de hausse des prix a simplement doublé, quel euphémisme, entre 1969 et 1974 pour passer de $4.5 \%$ à $10.7 \%$ au Canada, et de $5.4 \%$ à $11 \%$ aux États-Unis. Même si l'Amérique du nord fait figure d'îlot de stabilité dans un monde animé par une frénésie des prix qui n'est pas sans rappeler celle qui agitait l'Empire romain aux plus beaux jours de sa décadence, ces rythmes de hausse représentent sept fois celui du début des années 60 !

Et pourtant, pendant ces cinq années, les gouvernements ne se sont pas croisés les bras. Tout l'attirail traditionnel des politiques dites de stabilisation, politiques monétaire, fiscale, politique de revenus, discipline collective, contrôle, tout a été simultanément ou alternativement mis en oeuvre. Les États-Unis, le Canada, le Royaume-Uni ont appliqué des politiques restrictives de la demande. En France on a maintenu les mesures de stabilisation qui avaient été adoptées après la dévaluation du franc en 1969. Pour la première fois depuis la guerre, les Japonais ont jugé nécessaire d'accepter un ralentissement de l'activité économique. Le Danemark, la Finlande, les Pays-Bas, la Norvège, la Suède ont appliqué un contrôle sévère des prix. Les États-Unis comme chacun sait se sont résignés à adopter en 1971 des procédures de contrôle.

En bref, tous les gouvernements ont manifesté vers la fin des années 60 et au début de la décennie 70, leur volonté de lutter contre l'inflation, et ont pris à cet égard les mesures restrictives qui semblaient s'imposer.

Les résultats de leurs efforts s'apprécient à l'examen des courbes de prix mais aussi de chômage et de croissance. Depuis 1969 et pendant toute cette période le chômage s'est accentué en France, en Allemagne, en Italie, au Royaume-Uni, en Suède, ainsi qu'aux États-Unis et au Canada, malgré l'amélioration du niveau de l'emploi au cours des années 1972 et 1973.

Bien plus, la liaison croissance-emploi est elle-même menacée puisque malgré des taux de croissance qui pour l'ensemble des pays de 
l'OCDE, ont toujours été supérieurs à 3\% - atteignant même $6.3 \%$ en 1973 - la plupart des pays membres ont connu une augmentation du chômage. Avec $6.1 \%$ de croissance en 1971, les États-Unis enregistrent un taux de chômage de $5.6 \%$. Le Canada, la même année, voit son PNB augmenter de $5.8 \%$ avec un chômage de $6.3 \%$. La France enregistre son niveau record de chômage, depuis 1968 tout au moins, avec un taux de croissance de $5.5 . \%$ et une inflation de $5.9 \%$.

Enfin, en 1974, les prix s'envolent, le chômage s'alourdit, la croissance s'arrête. Avec une hausse des prix de $6 \%$ en termes annuels, les États-Unis ont enregistré au premier trimestre de 1975 une baisse de leur PNB de $10 \%$. Ceci ne s'était jamais vu depuis que des statistiques trimestrielles sont publiées, depuis 30 ans, et le chômage atteint $9 \%$. On ne compte pas moins de 14 millions de chômeurs dans les pays de l'OCDE à l'heure actuelle pour une diminution du PNB de $3 \%$ et une hausse des prix de $10 \%$.

Hier encore, il suffisait de quelques mesures de refroidissement pour que se calme la fièvre des prix et que la croissance reparte. Mais nous sommes vraiment dans un autre monde. Nous vivons dans une inflation, qui apparaît de plus en plus comme étant la fille naturelle de la croissance, sa seconde nature.

Dans ce contexte, l'analyse des politiques de l'après-guerre montre la nécessité d'avoir recours à de nouveaux instruments.

Mais qu'est-ce encore que l'inflation aujourd'hui ? C'est toujours un excès du flux de demande de biens et de services par rapport aux possibilités de l'offre qui provoque un mouvement auto-entretenu de hausse des prix, (accompagné selon que l'on se trouve en régime de taux de change fixes ou flexibles, d'un épuisement progressif des ressources nationales en réserves de change, ou d'une diminution de la valeur de la monnaie nationale).

Mais dès qu'il s'agit de déterminer la cause initiale expliquant pourquoi à un moment donné la demande devient excédentaire, il y a une multitude de raisons, apparemment toutes aussi bonnes les unes que les autres.

Des raisons monétaires d'abord: tout accroissement de l'offre de monnaie, pour une vitesse de circulation constante, à un taux supérieur à celui de la progression du produit réel, suscite, en l'absence de modifications du comportement des individus, un excédent de la demande 
globale exprimée en termes monétaires, et induit une hause des prix égale à l'écart entre les deux taux. Or depuis plusieurs années, on a enregistré dans la plupart des pays, un taux de croissance exceptionnel de la masse monétaire, bien supérieur au taux de croissance du Produit National Brut réel.

Des raisons internationales ensuite : toute économie en situation d'inflation et dont les prix des produits jouent le rôle de prix directeurs sur le marché international, exportent la hausse des prix en même temps que les dits produits aux économies qu'elle domine. La progression des liquidités internationales elles-même, n'a d'autres effets que ceux qui perturbent les économies nationales en cas d'offre excessive de monnaie. L'endettement du Tiers Monde est passé de 37 milliards de dollars en 1965 à 66 milliards en 1970 et 110 milliards en 1973. S'il est exact d'analyser ces mouvements de fonds comme un transfert d'encaisses épargnées - et par conséquent d'effet déflationniste - des pays industrialisés aux pays moins developpés, les pays du Tiers Monde ont dépensé ces fonds dans les économies développées pour y acquérir les biens et services qui leur font défaut. Comme par ailleurs les économies développées se trouvent pour tous les produits manufacturés en situation d'économie dominante vis-à-vis du Tiers Monde, ils y imposent leurs prix directeurs dont les niveaux s'élèvent d'autant plus que l'inflation y est plus forte. Non seulement l'inflation se généralise mais elle produit un effet de boomerang lorsque le Tiers Monde faisant état de ces augmentations réussit à y ajuster le cours des matières premières, nouveau facteur d'inflation par les coûts dans les pays développés, quels que soient leurs régimes de taux de change.

De 1960 à 1969, en dix ans, le volume des exportations mondiales a progressé de $100 \%$ alors que le montant des liquidités internationales n'augmentait que d'un peu plus de 20\%. De 1969 à 1973, ce sont les liquidités internationales qui cette fois ont plus que doublé - passant de 75 à 153 milliards de dollars - alors que les exportations en volume n'augmentaient que de $40 \%$. La comparaison des deux taux donne une idée de l'écart inflationniste. Il faut bien que l'équilibre se rétablisse, car les prix internationaux ne sont que les reflets ou les déterminants des prix nationaux.

Les pénuries sont également à l'origine des tensions inflationnistes. Elles consistent en une limitation potentielle de l'offre de biens et la hausse des prix doit intervenir pour annuler la demande excédentaire. Le cas typique est évidemment celui du pétrole dont l'origine tient à 
l'établissement d'un cartel par les gouvernements des pays producteurs. Les produits alimentaires sont un autre exemple où les pénuries cette fois sont dues à de mauvaises récoltes, et à l'absence d'un mécanisme international de partage des coûts d'entreposage.

La croissance elle-même est source d'inflation. Depuis un quart de siècle, le monde est dominé par la fièvre de la croissance. Or l'expansion ouvre à l'esprit une perspective enivrante, celle du jeu où tout le monde peut gagner, qui devient celle du jeu où tout le monde doit gagner, et si possible, de plus en plus et de plus en plus vite. L'abondance paraît comme une évidence aussi naturelle que l'air que l'on respire. De plus en plus on veut tout à la fois, le quantitatif et le qualitatif, ce qui revient à désirer les fruits de la croissance sans en accepter les impératifs. De plus, les nouvelles préférences privées ou collectives, telles que la protection de l'environnement, la réduction du temps de travail, la participation, la sécurité sociale, sont à la fois multiplicatrices de nécessités et réductrices de possibilités, ce qui les définit précisément comme des facteurs d'inflation. Comme cette croissance se développe dans un monde transparent dont les images sont transmises à des centaines de millions d'hommes à la fois, les convoitises sont exacerbées et l'abondance des uns crée une situation de pénurie subjective pour les autres. L'amélioration du niveau de vie devient un facteur d'irritation et de frustration croissante et un élément de dissension entre groupes sociaux. Dès lors, il n'est plus étonnant que dans cette course au plus, les mécanismes régulateurs des prix ne fonctionnent plus. Autrefois, un plan fiscal de redressement, assorti du battage psychologique approprié se traduisait par une ponction authentique du pouvoir d'achat et un ralentissement des tensions inflationnistes. Aujourd'hui l'accroissement de la pression fiscale est devenue elle-même inflationniste. La force collective des producteurs et des salariés est plus puissante que les mécanismes d'ajustement que tous refusent : une hausse des impôts appelle une hausse correspondante des salaires. L'inflation cause ou conséquence est alors acceptée comme un lubrifiant des mécanismes sociaux, aussi longtemps que le développement économique permet d'atténuer les aggressivités. Mais le pourra-t-il encore longtemps?

Il est certain par exemple que l'inflation sape les fondements mêmes de la croissance. Tout le développement économique, toute l'industrialisation repose sur la valorisation du futur par rapport au présent, sur l'idée que demain peut être meilleur qu'aujourd'hui. L'épargne est une privation de consommation qui, à ce titre, doit être rémunérée. Cette rémunération à son tour provient du rendement attendu des investissements. 
Or l'inflation rend le futur incertain, réduit les possibilités de trouver la rémunération nécessaire à l'épargne. Elle pénalise donc les épargnants qui "lâchent aujourd'hui une proie qui ne leur vaudra demain que l'ombre d'une ombre $\gg 1$. On peut admettre qu'avec l'augmentation générale du revenu, l'épargne représente aujourd'hui une privation d'utilités moindre qu'autrefois, et que par suite, on ait pu abaisser les taux réels de rendement du capital. Mais à l'heure actuelle la plupart des épargnes perçoivent un taux d'intérêt négatif compris selon les pays entre $-3 \%$ et $-12 \%$ l'an et inversement pour la valeur du capital à rembourser par l'emprunteur ${ }^{2}$. Il faut donc croire que l'inflation anticipée est au moins pour la moitié, inférieure à l'inflation observée. C'est l'apologie d'une monde où la rationalité économique exige des individus qu'ils consomment et s'endettent. Le «bon père de famille », aux placements sûrs à long terme est ridiculisé puisque les sacrifices en vue d'une sécurité ultérieure sont rendus vains. L'épargne est pénalisée, la prodigalité récompensée. L'homme qui vit en inflation n'a d'autres moyens pour se préserver de l'érosion de son pouvoir d'achat que de consommer dans l'immédiat la totalité de ses ressources. Les marchés manquent de capitaux. Les taux qu'il faut payer pour attirer l'épargne atteignent des niveaux incompatibles avec la rentabilité normale des entreprises, d'autant que l'incertitude interdit toute évaluation à moyen et long termes des projets d'investissements.

Tout investissement est un parti sur l'avenir et comporte pour l'entrepreneur un risque économique normal, dont le profit ou les dividendes pour l'actionnaire constituent la rémunération. Plus le risque est élevé, plus la rémunération attendue doit être élevée.

Quand il est possible d'en anticiper le taux, l'inflation contribue à élever le taux du risque pour l'entrepreneur et le taux d'intérêt nominal demandé par l'épargnant pour qu'il puisse obtenir de ses placements une rémunération réelle convenable. L'épargnant et l'entrepreneur s'opposent tous deux à assumer des risques additionnels, ils vont donc chercher la sécurité dans des placements plus liquides ou dans de placements de refuge qui ne sont pas particulièrement utiles à la croissance. Comme

1 Jacques RUEFF, L'âge de l'inflation, Paris, Études et documents Payot, 1963.

2 G. L. BACK, a calculé qu'aux États-Unis de 1946 à 1972, l'inflation non anticipée a entraîné un transfert des créditeurs aux débiteurs de l'ordre de 500 à 650 milliards de dollars. À l'heure actuelle, chaque point de pourcentage d'inflation non anticipée entraîne un transfert créditeur-débiteur de 40 à 45 milliards. La Banque de France estime que de 1960 à 1970 l'inflation a privé les ménages français de l'équivalent de 15 miliards de $\$$. 
d'autre part l'inflation est un phénomène dont les fluctuations s'inscrivent dans des périodes de court et moyen termes, elle contribue à réduire l'horizon économique des décisions. L'inflation par conséquent réduit les investissements à long terme. Or, ces investissements sont précisément ceux qui caractérisent le lancement d'un produit nouveau, d'une nouvelle technique, ceux qui conditionnent l'amélioration de la productivité et le progrès.

Mais si au surplus, comme dans la plupart des cas, le taux d'inflation ne peut être anticipé, prévu avec une reiative exactitude, alors le mécanisme même de l'investissement est compromis. Puisque ni l'épargnant, ni l'entrepreneur ne peuvent ajouter à leurs taux requis - de profit pour l'un, de rendement pour l'autre - un pourcentage représentant le rythme attendu de hausse des prix, l'inflation devient elle-même un risque qui vient se superposer au risque normal des affaires. Si malgré l'incertitude, le risque est pris, on se retrouve dans une situation analogue à la précédente, mais la décision d'investissement n'est plus qu'une fonction du tempérament plus ou moins sanguin d'individus irrationnels. La valeur d'un investissement, qu'il s'agisse d'outils, de machines, d'usines, est la résultante d'un flux de recettes attendues, actualisées au taux d'intérêt du marché financier. L'incertitude inflationniste rend aléatoire voire impraticable toute évaluation de ce genre. Les détenteurs de liquidités soucieux de minimiser les risques, préfereront se tourner vers d'autres emplois dont la valeur est en quelque sorte indexée à la hausse des prix. C'est la course dite «aux valeurs réelles », l'investissement, qui en fait n'en n'est plus un, consiste à acquérir des biens non reproductibles dont le fondement de la valeur n'est plus le rendement, mais la rareté. Terrains, immeubles, or, pierres précieuses, tableaux de maître, commodes Louis $\mathrm{XV}$, deviennent l'objet de toutes les convoitises et source des meilleurs profits.

L'épargne se tarit, l'investissement diminue, la productivité faute d'investissement se ralentit et finalement le chômage menace tandis que les titulaires de revenus nominaux fixes voient fondre la valeur réelle de leurs actifs. Seuls défendent leur niveau de vie ceux qui savent obtenir l'ajustement constant de leurs salaires, à des prix constamment croissants. «La fidélité à l'organe revendicateur plus que la fidélité à la tâche vaut sécurité et bien-être ${ }^{3}$. Le climat devient, de plus en plus conflictuel. De 1963 à 1970 sur les 2,000 accords de salaires qui ont été signés pendant cette période au Canada, 22.6\% l'ont été après moins de trois

3 Jacques RUEFF, op. cit. 
mois de négociations, $13.5 \%$ après dix mois ou plus. Entre 1971 et 1973, seulement $15 \%$ des 1,000 accords ont été signés en trois mois ou moins, et environ $20 \%$ ont demandé plus de 10 mois de négociations. En 1973, la proportion des accords qui ont été signés à la suite d'un arrêt de travail atteint $18 \%$ contre un taux d'environ $4 \%$ pendant les années 60 . On note également un accroissement du nombre de grèves intervenant avant l'expiration des conventions collectives, qui reflète la volonté des travailleurs d'obtenir un ajustement de leurs salaires à l'inflation. La réduction de la durée d'application des accords traduit également le rétrécissement de l'horizon économique. Entre 1962 et 1973, la proportion d'accords d'une durée d'un an n'a fait que diminuer passant de près de $20 \%$ à $12 \%$, alors que celle des accords de plus de deux ans est restée stable à environ $36 \%$. Pour le premier semestre de 1974, on a enregistré un profond renversement des tendances, la part des accords supérieurs à deux ans est tombée à $21 \%$ tandis que celle des accords d'une durée inférieure à un an est remontée à $19 \%$.

Enfin, puisque nous vivons dans un monde où l'interpénétration des économies nationales est de plus en plus marquée, il est évident que l'inflation ne vas pas sans perturber l'évolution des échanges extérieurs de chacun des partenaires. Les effets sont bien connus, en régime de taux de changes fixes comme en régime de changes flexibles. Dans le premier cas, l'élévation des prix des marchandises se traduit par un rétrécissement des marchés d'exportation, dans l'autre le taux de change s'ajuste, mais il faut exporter toujours davantage pour pouvoir obtenir un même volume de produits.

Cependant, c'est précisément parce que les nations sont de plus en plus interdépendantes au sein d'un même espace économique intégré que l'inflation y sévit partout. Or puisque tous les pays sont affectés, il n'y a plus de sanctions immédiates. Les déficits des balances des paiements et l'ardente obligation pour les gouvernements de préserver, ou de restaurer le cas échéant, les grands équilibres n'ont plus cours. Les contraintes ont disparu. Avec l'inflation généralisée à tous les pays, les gouvernements semblent avoir trouvé le secret du déficit sans douleur, car si d'aventure un des partenaires enregistre un déficit de ses paiements extérieurs, la même interdépendance suscite des mouvements compensatoires de capitaux.

Quant au secret de l'équilibre, il suffit que les pouvoirs publics interviennent de telle sorte que le taux d'augmentation des prix nationaux ne dépasse pas ceux des autres partenaires commerciaux pour que, de ce point de vue, l'inflation soit neutre. 
Il leur reste sur le plan interne à trouver les moyens de maîtriser un phénomène dont les taux actuels de progression sont en tout état de cause incompatibles avec l'existence même des fondements de la croissance. Les moyens traditionnels, fiscalité comprise, ont fait leurs preuves, ou plutôt ne l'ont pas fait. Les politiques d'après-guerre les ont largement utilisés avec les résultats que l'on sait. $\mathrm{Ni}$ la politique monétaire, trop brutale parce que trop efficace, ni la fiscalité classique ne semblent être adaptées au problème actuel de l'inflation. Néanmoins, si l'efficacité de la politique fiscale est à juste titre contestée, il est probable que la mise en oeuvre de nouvelles techniques en fera, à brève échéance, un moyen privilégié de lutte contre l'inflation.

Depuis la guerre, selon que Milton Friedman est plus à la mode que Keynes, ou inversement, la politique monétaire dispute à la politique fiscale le redoutable privilège de mener la bataille contre l'inflation. S'il y a unanimité sur un diagnostic qui perçoit l'inflation comme un déséquilibre entre le volume prévu des disponibilités nationales et celui de la dépense nationale, l'accord est loin d'être réalisé sur le genre de thérapeutique. Inspirée par un quantitativisme de principe qui pose que les prix et les revenus n'augmenteraient pas si l'on ne créait pas les liquidités correspondantes pour financer de telles augmentations, la ponction monétaire s'analyse comme un choc brutal s'exerçant non pas sur la demande ou l'offre globale mais sur les liquidités monétaires que l'on soumet à une brusque et rigoureuse contraction. Cette déflation de la quantité de monnaie en circulation peut exercer une forte contrainte sur le niveau des prix, et l'obliger à baisser. Elle entraîne une diminution des actifs liquides ou financiers détenus pas les agents économiques, dans la mesure où ceux-ci doivent les mobiliser pour financer leurs transactions. Comme d'autre part, les individus déterminent le niveau de leurs dépenses de manière à maintenir constante la structure de leur patrimoine actif et passif, ils vont avoir tendance à prélever sur leurs revenus courants les liquidités nécessaires à la reconstitution de leurs avoirs, et contribuer par conséquent à réduire la demande globale. Ainsi la politique monétaire a l'avantage d'être plus générale qu'une politique fiscale de régulation de la demande, puisqu'elle affecte à la fois les prix, les revenus, les dépenses de consommation, et les investissements par le biais des taux d'intérêt et des restrictions quantitatives de crédit.

C'est bien parce qu'elle est globale qu'elle est contestée. En frappant toutes les composantes de la demande elle risque de susciter la dépression en limitant les investissements. Par ailleurs, elle doit être brutale pour 
pouvoir enrayer l'inflation par les coûts car elle ne peut parvenir à stopper l'inflation salariale qu'en induisant une baisse de la production. $\mathrm{Si}$, d'autre part, les tensions inflationnistes qu'elle se propose de combattre n'ont pas un caractère accidentel, elles réapparaîtront sitôt les encaisses reconstituées. La politique fiscale d'inspiration Keynésienne apparaît comme devant être plus sélective, puisqu'elle ne s'applique qu'à la demande et peut être modulée selon qu'il s'agit de consommation ou d'investissement. Appliquant le principe de la demande effective, il s'agit de prélever par l'augmentation de l'impôt ou par la réduction des dépenses publiques, une part du revenu nominal, telle que la dépense du revenu disponible après impôt, corresponde au total des ressources disponibles. Cette politique peut être mise en oeuvre de deux façons différentes. L'action déflationniste peut résulter d'une certaine structure fiscale à peu près invariable dans le temps et dont les effets de stabilisation sont automatiques. À mesure que croissent les revenus nominaux, l'application de l'échelle progressive des taux d'imposition se traduit par un accroissement automatique du prélevement fiscal plus que proportionnel à l'accroissement des revenus, contribuant ainsi à ralentir la demande globale. Cette politique, dite de flexibilité interne, n'est le plus souvent considérée que comme un accessoire d'une politique globale, qui accorde la préférence aux mécanismes monétaires dans la recherche de la stabilité, et qui répugne à confier à l'action discrétionnaire des pouvoirs publics un trop large pouvoir d'intervention. En fait la flexibilité interne peut jouer un rôle d'amortisseur de tensions, mais elle ne peut à elle seule assurer la régulation de l'activité économique.

Dans ces conditions, on préfère manier délibérément les taux d'imposition de manière à ajuster le montant du prélèvement fiscal à l'évolution des flux de revenus et de dépenses prévus. La politique fiscale devient une politique de compensation active. Elle permet d'autre part aux pouvoirs publics d'accumuler des réserves pendant la phase d'expansion inflationniste qu'ils pourront dépenser pour soutenir l'activité économique pendant la phase de ralentissement du cycle. C'est la technique dite du Budget conjoncturel ou du Fonds d'action conjoncturel.

Cependant quelles que soient les techniques fiscales utilisées - imposition directe avec relèvement de taux, échelle de taux fortement progressive selon les tranches de revenu, ou impôts indirects - l'effet déflationniste recherché par le biais du prélèvement fiscal n'est pas toujours atteint. Dans une conjoncture où l'inflation apparaît de plus en plus 
comme une donnée permanente, la fiscalité peut au contraire contribuer à alimenter et renforcer les tensions inflationnistes.

Il en est ainsi des impôts indirects. Puisqu'ils sont perçus sous la forme de taxes de vente, ils semblent à première vue posséder une double efficacité. Il se traduisent d'une part par un prélèvement de pouvoir d'achat au moment de la dépense, et par conséquent n'affectent pas l'épargne, et d'autre part, par une hausse de prix, qui en bonne logique devrait constituer un frein à la consommation. Or en période d'inflation, l'effet de freinage de la consommation est illusoire, car les élasticités-revenus comme les élasticités d'anticipation des prix jouent en sens inverse. On accroît au contraire le mal que l'on prétend combattre dans la mesure où les produits intermédiaires sont frappés, car l'augmentation des prix à laquelle donne lieu l'élévation des impôts, augmente les coûts de production et se répercute sur les produits finals.

Le professeur Auld de l'Université de Guelph en Ontario a montré qu'au Canada en 1949, sur le total des achats de biens de consommation faisant l'objet d'une taxation indirecte, $31 \%$ des dépenses correspondaient au paiement de l'impôt. Pendant les 10 années suivantes, ce taux s'est élevé pour atteindre $35 \%$ en 1960 , puis 10 ans plus tard, en $197046 \%$. Il est évident que le gouvernement est pour une part responsable de la hausse de l'indice des prix à la consommation.

Quant à l'impôt direct, en frappant le revenu il frappe autant les dépenses que l'épargne et en limite la formation pour un revenu brut donné. À mesure que l'on s'élève dans la hiérarchie des revenus, l'impôt est davantage financé par l'épargne que par une réduction de la consommation. Dans le bas de l'échelle des revenus, le niveau de consommation étant jugé incompressible, l'impôt - et notamment lorsqu'il s'agit d'une augmentation devant être provisoire - a tendance à être financé par la liquidation totale ou partielle des patrimoines, ou l'endettement. Dans ces conditions l'imposition suscite une désépargne.

Certes à l'échelle d'une nation, il ne peut y avoir désépargne collective puisque ce qui est vendu par les uns est acheté par les autres. Mais ces arbitrages entre patrimoines modifient la structure du capital national et augmentent la vitesse de circulation des encaisses, que ces encaisses alimentent les transactions en capital où qu'elles passent dans le circuit des dépenses courantes. La quantité de monnaie aura tendance à augmenter, elle variera en définitive en fonction de la réaction des 
banques, qui, selon la politique monétaire en vigueur, transformeront ou non ces épargnes en offre additionnelle de monnaie. Il ne peut donc y avoir de politique fiscale anti-inflationniste efficace sans politique monétaire.

Par ailleurs l'impôt direct réduit le rendement de l'épargne et celui des placements, il peut soit décourager le contribuable, soit le pousser à prendre plus de risque. En fait, en augmentant le prix du risque, ou en réduisant le rendement net d'un placement comportant un risque donné, il réduit l'avantage comparé des placements risqués par rapport aux autres, et pousse l'épargnant à leur substituer des emplois moins risqués. Ainsi même si l'épargne courante en volume n'est pas modifiée, la structure des placements peut l'être. Ceci contribue d'une façon générale à rendre l'économie plus liquide, les contribuables donnant la préférence aux encaisses et placements surs qu'il est relativement plus facile de liquider. L'économie devient ainsi plus sensible aux pressions inflationnistes puisque son pouvoir d'achat potentiel constitué par des patrimoines liquides accroît l'autonomie de dépenses. L'intensité de cet effet dépend, là encore, de la politique monétaire nationale. On ne peut manquer par ailleurs, d'être frappé de la similitude de ces effets avec ceux de l'inflation sur le choix des placements. Ainsi, l'effet déflationniste du prélèvement fiscal est loin d'être aussi effectif, et aussi mécanique qu'il ne le paraît.

En fait, au delà d'un certain seuil d'inflation, toute politique visant à réduire la demande risque d'aggraver les tendances inflationnistes au lieu de les modérer, non seulement sous l'action des mécanismes économiques, mais aussi des facteurs institutionnels et politiques sans rapport avec la régulation de la demande proprement dite. Il en est ainsi des politiques de redistribution qui consistent à prélever aux titulaires de hauts revenus, des liquidités destinées à l'épargne afin de les répartir entre les titulaires de bas revenus à forte propension à consommer. En transformant une épargne potentielle en consommation, on contribue ainsi à renforcer les pressions de la demande. D'autre part, lorsque par souci d'équité, les gouvernements indexent les transferts et les revenus les plus faibles, ou augmentent les paiements de sécurité sociale, ils contribuent du même coup à réduire l'efficacité des mesures anti-inflationnistes.

En période d'inflation, les travailleurs revendiquent des accroissements de salaires suffisamment élevés pour préserver leur revenu réel 
disponible, pour maintenir le pouvoir d'achat de leurs salaires après impôts. Or, puisque les structures fiscales sont à taux progressifs, le taux marginal excède le taux moyen. Le maintien d'un même revenu disponible exige un accroissement des rémunérations, supérieur à celui de la hausse des prix. En effet, un accroissement du revenu nominal brut, de $8 \%$ par exemple, fait passer son titulaire dans une tranche supérieure de revenu où le taux d'imposition est plus élevé. Il subira un accroissement du prélèvement fiscal plus que proportionnel à son accroissement de revenu. Or, si ce $8 \%$ de croissance ne correspond qu'à la hausse des prix, son revenu réel disponible après impôts aura diminué. Il existe donc une inter-action entre inflation et progressivité de l'impôt. La progressivité de l'impôt suscite des revendications supérieures à la hausse des prix qui suscitent à leur tour de nouveaux accroissements de prélèvement fiscal. Tel est le schéma de la «tax-push inflation » sur laquelle le Conseil économique du Canada a attiré l'attention des pouvoirs publics dans son Exposé annuel de 1972.

Une telle analyse suppose une certaine part d'illusion fiscale. Lorsqu'ils prennent en considération dans leurs revendications de salaires, les prélèvements fiscaux plus élevés à cause de l'inflation, tout se passe comme si les travailleurs oubliaient les bénéfices qu'ils retirent des dépenses publiques que ces accroissements réels d'impôts permettent de financer.

Ainsi, entre une politique monétaire nécessaire mais d'une brutalité intolérable, et une politique fiscale plus souple mais d'une efficacité douteuse voire même inflationnistes, les outils traditionnels de la politique économique ne semblent guère adéquats pour nous permettre de gérer l'inflation.

Or, toutes ces politiques ont entre elles une caractéristique commune : elles traduisent une volonté d'intervenir pour modifier les emplois $\mathrm{du}$ revenu, mais n'agissent guère au niveau de leur formation. Puisque les revenus des uns constituent nécessairement les coûts des autres, et que chacun est devenu un parfait modèle de rationalité en ajustant en permanence son revenu à ses coûts, toute intervention au niveau de la formation des revenus présenterait ce double avantage de limiter les augmentations excessives de revenu, donc de demande, et de contrôler les coûts. Ajoutons qu'il deviendrait inutile de poursuivre plus longtemps le débat entre partisans d'une explication de l'inflation par les coûts et partisans d'une explication par la demande. 
De telles politiques ont été mises en oeuvre par le recours à d'autres techniques que les techniques fiscales. Il s'agit des politiques de prix et de revenus. D'une façon générale, ces politiques s'efforcent d'agir sur la formation des revenus plutôt que sur les ressources déjà perçues. Elles s'attachent au gain à venir plutôt qu'à la propriété acquise, elles sont indicatives ou contractuelles plutôt qu'impératives.

Appliquée avec quelque succès aux Pays-Bas dans les années 50, la politique des revenus qui repose avant tout sur la concertation des partenaires sociaux, a été tentée sans succès en Grande Bretagne dans les années 1961-1966 où elle s'est soldée par un blocage impératif des prix et des salaires. La France a également cherché à mettre en place de tels mécanismes de concertation sans davantage de succès au milieu des années 60. D'une façon générale, le manque de moyens coercitifs, qui remettraient du même coup le mécanisme de concertation en question, ne permet pas à de telles politiques de remporter des succès durables et d'être érigées en institution permanente.

Quant au contrôle des prix et des salaires, on connait son efficacité. S'il parvient à limiter les hausses pendant une courte durée, le problème se pose immanquablement, de savoir comment ménager le retour à un régime plus normal sans laisser exploser les revendications qui se seront accumulées pendant la durée du blocage. S'il s'agit d'un système plus souple destiné à être permanent, il devient rapidement contourné et sombre dans l'inefficacité.

Et pourtant, c'est incontestablement dans cette nouvelle voie qui consiste à intervenir au niveau de la formation des revenus, que la fiscalité est non seulement appelée à se renouveler, mais qu'elle contribuera à doter les pouvoirs publics d'instruments adaptés à l'âge de l'inflation. Déjà les chemins dans lesquels il convient de s'engager ont été tracés par les praticiens autant que par les théoriciens. C'est ainsi que la France a mis au point en janvier 1975 un «prélèvement conjoncturel $»$ qui est l'illustration des nouvelles techniques fiscales anti-inflationnistes proposées entre autres, par le professeur Weintraub.

L'objectif fondamental de la taxe conjoncturelle française est d'inciter les entreprises qui obtiennent des gains de productivité supérieurs à la moyenne nationale, à les répercuter aux utilisateurs finals sous la forme d'une baisse de leurs prix de vente. Le mécanisme en est simple et peut s'expliquer de la façon suivante. Si pour l'année 1975, le volume de la 
production nationale doit augmenter de $5 \%$ et le niveau général des prix de $10 \%$, il est dans ces conditions tout à fait normal que la marge brute d'une entreprise progresse d'environ 15\% par rapport à l'année 1974. Mais si la croissance de cette marge est supérieure à la norme de $15 \%$, disons $25 \%$, l'entreprise devra justifier cette croissance supplémentaire de $10 \%$. Si cet excès de croissance n'est que le reflet d'une augmentation de la marge brute due à une augmentation du nombre d'heures travaillées, ou à un accroissement des capacités de production, il ne sera pas taxé. $\mathrm{Si}$ au contraire rien de tel ne le justifie, l'administration fiscale présume qu'il a été obtenu, soit par une augmentation des prix de vente supérieure à la hausse du niveau général des prix, soit par un accroissement de productivité supérieur à la norme nationale non répercuté par une baisse de prix. Dans les deux cas cet excès est taxé au taux de $331 / 2 \%$. Ce prélèvement exceptionnel est destiné à être remboursé aux entreprises lorsqu'il sera supprimé, c'est-à-dire lorsque la progression de l'indice général des prix restera inférieure à $0.5 \%$ pendant trois mois consécutifs.

Un tel mécanisme est l'application d'une technique imaginée par le professeur Weintraub dans un article publié en 1970. Celui-ci constate que depuis 20 ans, les accroissements annuels de productivité aux ÉtatsUnis et en Grande-Bretagne ont été en moyenne de 3\%. Si l'on accepte une hausse des prix de l'ordre de $2 \%$ par an, il est convenable d'envisager des augmentations annuelles de salaires de l'ordre de 5\%, soit un doublement de revenu tous les 15 ans à travail égal. Dès lors il suffit de pénaliser les entreprises qui accordent des augmentations de rémunérations d'une année sur l'autre supérieures à $5 \%$. Si l'an dernier, le salaire moyen payé aux employés de l'entreprise était de $\$ 10,000$ et si pour cette année il excède $\$ 10,500$, alors l'entreprise devra payer une surtaxe anti-inflationniste sous la forme d'une majoration de son impôt habituel. Si elle est assujettie à un impôt sur les bénéfices de $40 \%$ et si l'accroissement du salaire moyen par rapport à l'année précédente dépasse les $5 \%$ mais est inférieur à $8 \%$, une surtaxe de $10 \%$ est ajoutée, et son taux d'imposition devient $50 \%$ au lieu de $40 \%$. Pour des accroissements de salaires compris entre 8 et $12 \%$ la surtaxe pourrait être de $15 \%$ et ainsi de suite, de façon à ce qu'elle soit progressive et pénalise d'autant plus lourdement les entreprises que celles-ci participent plus activement au développement de l'inflation. Un tel système peut être généralisé en l'appliquant non seulement aux entreprises mais à tous les agents économiques, par le biais d'une surtaxe fixe ou progressive sur le revenu personnel. Dans le cas d'une surtaxe à taux progressifs, l'échelle pourrait être déterminée selon l'importance des excédents de revenu par: rapport 
à la norme, ou mieux, selon le niveau absolu des revenus de chacun, de manière à renforcer le caractère redistributif de l'impôt classique. Une telle formule, plus générale que la proposition du professeur Weintraub, a ma préférence, dans la mesure où je ne suis pas sûr, contrairement à Sydney Weintraub, que les entreprises ne repercuteraient pas dans leurs prix de vente les effets de la surtaxe qu'elles doivent acquitter lorsqu'elles consentent des augmentations excessives de salaires. Les vertus de la concurrence ne sont pas monnaie courante dans des structures oligopolistiques, et deux précautions valent mieux qu'une.

Aux termes de 25 ans de croissance quasiment ininterrompue et d'applications devenues classiques de politiques de régulation, l'inflation est plus vigoureuse, plus ardente que jamais. Les causes en sont devenues multiples à un tel point que leur identification a perdu tout intérêt pratique. L'expérience récente nous enseigne qu'il faut cesser de vouloir rompre des lances contre des moulins à vent, au risque de devoir porter le deuil de notre société. Il faut découvrir et appliquer de nouvelle techniques de gestion de la demande globale et réussir à contenir l'inflation sinon nous serons contraints, soit de réduire la croissance aux niveaux antérieurs à ceux des quinze dernières années, soit d'abandonner les espoirs d'un partage plus équitable des richesses dans le monde.

\section{Inflation and Fiscal Policy}

Postwar economic history can be seen as an unremitting effort towards that three-pronged objective (growth, full employment and price stability) constantly refined over the years, but never sufficiently perfected to permit successful achievement on a permanent basis.

It was realized very soon during those years that, while two out of the three objectives were reached quite easily, to close the triangle was a puzzle not readily resolved, and that especially so since the British economist Phillips plotted in 1958 a few curves which have become famous, apparently proving the existence of a close and stable inverse relationship between the rate of price increase and the level of unmployment.

The triangle was therefore transformed, and in the 1960s the rule became based on three times 3 per cent : a minimum growth rate of 3 per cent in volume could readily be made to coincide with a maximum price increase of 3 per cent and an unemployment rate of the order of 3 per cent. Until recently, with the exception of the United States and Canada, where the unemployment rate tended to exceed the 3 per cent but where prices were generally more stable, most OECD 
countries conformed reasonably well as new representatives of the triangle. Inflation existed, but it was merely creeping. In the second half of the 1960s, matters deteriorated and the rule of three times three fell apart. In June 1971, the experts of the OECD Economic Policy Committee wrote : "It has been recognized, in fact, that there is no simple or constant relationship between the rates of price increase, on the one hand, and the degrees of demand pressures, the rate of growth and the levels of unemployment, on the other. The answer has been given by an explosion of prices that seems to persist despite a sharp rise in unemployment and a slowing down of growth. There is no longer any triangle; our policies have lost their foundation. Towards the end of the 1960s and the beginning of the 1970s, all governments expressed their will to fight inflation and took the restrictive measures that were felt necessary to that end. As a result of these efforts, there are not, at the present time, less than 14 million unemployed workers in the OECD countries, where an average of 3 per cent decrease of GNP and 10 per cent price increase were recorded in 1974. In this context the analysis of postwar policies reveals an obvious need to use new instruments.

What is this inflation of today? It is still an exceed demand for goods and services in relation to supply, leading to a self-sustained price increase - which is accompanied by either a progressive exhaustion of reserves of foreign exchange or a decrease in the value of the national currency according to whether the exchange rate is fixed or flexible. However, as to the underlying reason why at a given moment there is excess demand, several explanations exist, each of which is apparently as good as the others. First, the monetary explanation : assuming a constant rate of circulation in the stock of money, any increase in the money supply, over and above the rate of gain in real production, inevitably gives rise (unless there is some change in individual behavior) to an excess of total demand expressed in nominal terms, which leads to a price increase equal to the gap between the two rates. Then there are the international explanations: where the prices of certain products are rising, for one reason or another, countries that are price leaders on international market for those products export inflation to other nations, thus spreading the infection. Shortages are also at the origin of inflationary strains. They involve a potential limitation in the supply of goods, so that an increase in prices must occur in order to cancel the excess demand. Furthermore one must remember that growth itself is a source of inflation. Moreover, the new private or collective preferences, such as environmental conservation, shorter work periods, management participation, and social security, both multiply needs and reduce potential supply so that they become inflation factors.

It is not longer surprising, then, that in this rush for more the price-regulating mechanisms have stopped working. Formerly, a fiscal plan for readjustment, accompanied by the appropriate psychological conditioning, resulted in a genuine lowering of purchasing power and a slowdown of inflationary pressures. Inflation as a cause or consequence is then accepted as a lubricant of social mechanisms for as long as economic development helps to mitigate aggressive feelings. But how long will it play that role? It is clear, for instance, that inflation undermines the very foundations of growth, since inflation clouds the future with uncertainty, reduces the chances of finding the necessary money for saving, and therefore 
penalizes savers who «today forgo the substance in order to find tomorrow only the shadow of a shadow». Saving is penalized, prodigality is rewarded. Any investment is a bet on the future and implies for the entrepreneur a normal economic risk, the reward for which is the profits or dividends accruing to the investor. When its rate can be forecast, inflation serves to increase the risk for the entrepreneur and thus raise the nominal interest rate required by the saver in order that he may obtain from his investments a reasonable real return. On the other hand, since inflationary movements take place within short- and medium-term time periods, they lower the economic horizon for decision-making. Therefore, inflation reduces long-term investments, and these are precisely those investments that are associated with the introduction of a new product or a new technique ; they also are those that command productivity improvement and progress. But if, in addition, as is the case most of the time, the rate of inflation cannot be fully anticipated then the very mechanism of investment is jeopardized. The value of an investment is a flow of expected returns discounted at the interest rate prevailing on the financial market. Yet any assessment of this type becomes risky and even impossible due to the uncertainty associated with inflation. Savings dry up, investments decrease, productivity slows down for lack of investments, and finally unemployment threatens, while recipients of fixed money incomes experience a decrease in the real value of their assets.

The climate becomes more and more antagonistic. Negotiations get longer, the number of strikes occuring before collective agreements increase, this reflects the will of workers to have their wages indexed to inflation. Finally, since we are living in a world where national economies are increasingly intermingled, it is obvious that inflation disturbs the development of the external trade of all partners. However, it is precisely because nations are increasingly interdependent within a single integrated world economy that inflation is everywhere. Governments then must find other ways to achieve internal balance and control a phenomenon which at present rates of increase undermine the very basis of growth. The traditional instruments, including tax policies, have played their part - or rather have failed to do so, because postwar governments have used them widely with the results we know. Neither monetary policy, which is too brutal or effective to be used fully, nor the traditional fiscal measures, seem to be able to cope with the current inflation problem.

Nevertheless, even though the efficiency of fiscal policy is questioned, and rightly so, it is likely that the implementation of new techniques will make it before long a preferred means for fighting inflation. Monetary policy has the advantage of having a wider impact than fiscal policy as a means of regulating demand, since it influences prices, incomes, consumer expenditures, and investments altogether through interest rates and quantitative credit restrictions. It is precisely because of its very wide impact that monetary policy is questioned. By acting on all demand components including investments it is powerful enough to create unemployment. Indeed, it must strike hard to stop cost-push inflation, and induce that reduction in output that will put pressure on costs. For this reason, it is preferable deliberately to manage tax rates so as to adjust the amount of tax increases to the evolution of anticipated income and expenditure flows. Fiscal 
policy then becomes an active contracyclical instrument. However, whatever fiscal techniques are used the deflationary effect sought through fiscal action is not always attained. In an economic climate in which inflation is becoming more and more a permanent element, the tax system can instead tend to feed and reinforce inflationary pressures.

Between a monetary policy that is necessary but intolerably blunt and fiscal policy that is more flexible but of doubtful efficiency - and perhaps even a contributor to inflationary pressures - the traditional instruments of economic policy do not seem very adequate to enable us to control inflation. Nevertheless, all these policies share a common feature : they have an impact on the utilization of income but hardly any on the determination of income. Since the incomes of some people necessarily constitute costs for others, any intervention at the level of income formation would present the double advantage of limiting excessive increases in income and thereby controlling costs. Such policies have been developed by resorting to devices other than fiscal ones such as prices and incomes policies. Generally, these policies strive to bear upon the formation of incomes rather than on their potential uses; they are related to future rather than past gains, they are indicative or contractual rather than imperative. Generally, the lack of coercive measures which would bring into question the concertation mechanism does not lead to lasting success and cannot be institutionalized. With respect to wage and price controls, we are well aware of their merits and demerits. If it is a more flexible system that is conceived to be permanent, it then becomes rapidly distorted and sinks into inefficiency. Nevertheless, it is undeniably in this new direction, involving an intervention at the level of income formation, that the fiscal system will be called upon to move in order to help provide public authorities with instruments appropriate to the era of inflation. Already the avenues in which it needs to move have been laid out by practitioners and theoricians alike. To give an example, the French government introduced in January 1975 a stabilization levy which is an application of the anti-inflationary fiscal techniques suggested by Professor Weintraub among others. Such a system can be generalized by applying it not only to business firms but to all economic agents by way of a fixed or progressive surtax on excess incomes. In the case of progressive rates, the scale could be determined by reference to the size of the increase over and above the stated quideline or perhaps to the income level itself so as to reinforce the redistributive nature of the system. Such a formula, more general than the suggestion advanced by Professor Weintraub, would be my preference since I suspect that business firms might be tempted to shift the burden of the tax to their customers. After 25 years of almost uninterrupted ecoonmic growth supported by a refined and classic system of economic regulation, inflation is more vigorous and entrenched than ever. Its causes are so wideranging that their identification has lost practical interest. Recent experience has shown that we must stop tilting at windmills lest we end by mourning the ruin of our society. We must find and apply new techniques of overall demand management and succeed in checking inflation; otherwise, we will have no choice but to reduce growth to levels below those we have enjoyed over the past 25 years or to forsake our hopes for a more equitable sharing of wealth in the world. 